\title{
Die Ballade vom kranken Gesundheitssystem -
} 2. Akt

François Gilliet

Korrespondenz: Dr. med. François Gilliet Via Camminata 6 CH-6500 Bellinzona Tel. 0918256333 Fax 0918259385 studio.gilliet@bluewin.ch
Ein Jahr danach - one year after: Der erste Akt dieser Ballade ist vor einem Jahr verfasst worden. Ich habe auf die Publikation sehr viele Zuschriften erhalten, weitaus die meisten lobend, kaum solche mit Kritik, keine einzige, die mich der Unwahrheit bezichtigt hätte. Das BAG hat nicht reagiert und ist mit sich selbst beschäftigt, und von den andern, direkt beteiligten und angeschriebenen Persönlichkeiten (Suva und santésuisse) hat niemand Stellung bezogen, ausser, dass mein Aufsatz der eines alten Arztes sei, was ich wirklich nicht abstreite. Eigentlich wollte ich es dabei bewenden lassen. Einige Vorfälle und besondere Ermunterungen haben mich doch dazu bewogen, in dieser Fortsetzung noch einmal über unser Gesundheitswesen aus meiner zugegeben etwas engen, dafür aber essentiell wichtigen, auf den medizinischen Alltag und den Patienten bezogenen Warte zu berichten:

\section{Zweiter Akt}

Vieles ist geschehen, geändert hat sich nicht viel: Man spricht nur vom Sparen. Einsparungen sind jedoch keine zu verzeichnen. Die direkte, ärztliche Versorgung der Bevölkerung im Alltag ist anders geworden, jedoch nicht besser, sondern nur besser kontrolliert und bürokratisiert.

\section{Der Patient im neuen Gesundheitswesen}

Die Patienten müssen länger warten, bis sie zum Arzt gelangen [1]. Die Arzt-Patienten-Beziehung ist unpersönlicher geworden. In der Dermatologie und Allergologie laufen mehr Patienten mit Wahrscheinlichkeitsdiagnosen herum und sind verunsichert. So werden Hautbiopsien mit histologischen Untersuchungen weniger verordnet, um die Kosten tief zu halten. Stützt man sich vorerst einmal auf das Resultat von Probebehandlungen, steigen die Kosten aber sekundär und die Patienten wissen nicht mehr, woran sie sind.

Die Notfallstationen behandeln jetzt auch gewöhnliche Fälle, für die Patienten (besonders abends) eine äussert bequeme, aber nicht unbedingt gute Alternative. Die dort arbeitenden, meist jungen Ärzte sind häufig überfordert und reagieren gezwungenermassen mehr als früher unsicher und zögerlich aus Furcht vor Anfechtungen mit gerichtlichen Folgen. Ohne ausgedehntes Labor, Röntgen und apparative Bildge-

\section{Zusammenfassung des ersten Aktes}

Im ersten Akt (Schweiz Ärztezeitung. 2006; 87[17]:719-21) habe ich aufgezeichnet, wie sich unser Gesundheitswesen unter dem Zwang zum Sparen, zusätzlich mit der politischen Zielsetzung der Globalisierung (und auch der Transparenz) in der Medizin, gewandelt hat. Den Ärzten wird ein bedeutender Teil der Schuld am Versagen des alten Systems zugeschoben. Die Konfliktbildung dieser Ballade entsteht hauptsächlich wegen des Nichterreichens des Ziels vor allem des Sparens, wobei offensichtlich die gewollte und gelenkte Unterdrückung des Einflusses der Ärzte dem Staat ebenso wichtig ist. Hier ist allerdings eine klare Bilanz unmöglich. Die zunehmende Verstaatlichung unseres Gesundheitswesens wird langsam zum Hauptmotiv für die repressiven Veränderungen, und tritt immer offener in Erscheinung. Sie hat sich inzwischen schon weitgehend etabliert.

bung geschieht da nichts mehr, eine eher teure Medizin, also das Gegenteil des ursprünglich Gewollten. Vom privaten Hausarzt versorgte Notfälle sind deshalb im Durchschnitt auch nur halb so teuer wie die der Notfallstation [2]. Der «Pronto soccorso» ist also nicht die Lösung. In gewissen Situationen (z.B. bei Notfällen) sind also autoritäre, auf Erfahrung beruhende Entscheidungen aber auch heute immer noch gefragt und unersetzbar.

Auch in der alltäglichen Privatpraxis hat sich für den Patienten einiges geändert. Dinge, die früher selbstverständlich schienen, können aus Personal- oder Zeitmangel nicht mehr durchgeführt werden (TARMED heisst ja Medizin auf Zeit, wobei seltsamerweise nicht die Zeit, sondern die Medizin zum dehnbaren Begriff geworden ist). Man hilft den Patienten nicht mehr (man kümmert sich nicht mehr), sondern drückt ihnen Flug- oder Merkblätter in die Hand und gibt ihnen Internetadressen, dass sie sich selbst helfen sollen. Aber so entstehen auch Fehlinterpretationen, die sich unwidersprochen verbreiten können. Patientenkontrollen erfolgen spärlicher 
oder überhaupt nicht mehr, um den Falldurchschnitt nicht zu gefährden. Die Mittelmässigkeit der Schweizer Medizin hat sich damit fest etabliert.

\section{Der Arzt im neuen Gesundheitswesen}

Auch das Ansehen des Arztberufes hat sich gewandelt. Dennoch scheint der Ausspruch: «die Ärzte sollen sich in Zukunft in ihrem Beruf weniger bestätigen und ihn nicht (mehr) als Berufung empfinden, den Arztberuf vielmehr als Rolle und nicht als Identität betrachten» («Medical Tribune», Juni 2006), kaum mehr als ein politischer Slogan zur Rechtfertigung eines nicht funktionierenden Systems zu sein. Sind es nicht die Patienten, die ein solches Arztbild schaffen oder suchen und nicht die Ärzte? Deshalb hat die Rede von Prof. Marco Mumenthaler anlässlich der letztjährigen medizinischen Staatsexamensfeiern in Zürich an Aktualität nur gewonnen [3]. Unser Gesundheitswesen braucht überzeugte und nicht frustrierte Ärzte, die ebensogut als Taxifahrer ihr Geld verdienen könnten. Der Beruf des Mediziners hingegen sollte trotz allem auch heute noch nicht nur gelernt und praktiziert, sondern gelebt und erlebt werden. Wenn es um finanzielle Einschränkungen geht, erklären einige Politiker jedoch ohne Hemmungen, die Ärzte hätten bei der Berufswahl ja gewusst, auf was sie sich einlassen (Druck und lange Arbeitszeit, heute auch mehr finanzielle Probleme), und müssten somit motiviert (eben berufen) sein. Ein Student muss ja ein Traumtänzer sein, wenn er mit solchen Auflagen überhaupt noch Medizin studiert. Der freie Arztberuf gehört schon seit Jahren der Vergangenheit an. Das Einkommen des Arztes wird limitiert, zensuriert und zurückgesetzt auf dasjenige eines Beamten [4], er trägt jedoch noch immer das volle finanzielle Risiko eines Unternehmers. Aber nicht nur der Arztberuf ist anders geworden, auch die Ärzte werden sich ändern. Die Auslese der jüngeren Generation wird weniger humanistisch (menschlich?) denkende, sondern notwendigerweise mehr berechnende (auch mehr streitbare) Mediziner hervorbringen. Auch in der Medizin wird die Polarisierung zunehmen.

\section{Die Rolle der Behörden im neuen Gesundheitswesen}

Die Rolle des Staates sollte es sein und bleiben, Garant dafür zu sein, dass jedem seiner Bürger der Zugang zur besten, verfügbaren Medizin ermöglicht wird. Im Moment steht jedoch nicht dieses Recht für den Patienten im Vordergrund, sondern der Staat strebt nur nach der Vergrösserung seiner eigenen Macht.
Die Globalisierung in der Medizin wird uns in Zukunft zwingen, schwere, weittragende Entscheidungen treffen zu müssen, welche Behandlungsformen angebracht und sinnvoll sind und auch, ob eine Therapie möglich ist oder nicht: Die (leider irreale) politische Vorstellung, alle Menschen gleich (gut) behandeln zu können, muss einer mehr wirklichkeitsnahen, differenzierteren und immer wieder neu abzuwägenden Beurteilung weichen. Die Folgerung ist, dass die gleiche Medizin für alle nie möglich sein wird, da die Vorbedingungen nicht gleich sein werden. Dass in Zukunft - wie schon vorgesehen - globale medizinischen Entscheidungen im kleineren Rahmen auch bei uns (man denke nur an die Altersmedizin bei unserer zunehmenden Überalterung) nicht von Ärzten, sondern von staatlich eingesetzten Sanitätsfachleuten getroffen werden können, wage ich zu bezweifeln. Im Einzelfall steht dann die geeignete Person doch nicht zur Verfügung und es werden unsinnige Beurteilungen abgegeben, wie ich es schon im ersten Akt unter «wahre Geschichten» (Gürtelrose) dokumentiert habe. Auch das Berufsgeheimnis könnte nicht mehr aufrechterhalten werden. Bei den anstehenden Problemen müssen wir Ärzte das Zepter in der Hand behalten und nicht lediglich technisch, aber nicht medizinisch ausgebildeten, jungen und noch unerfahrenen, aber umso überzeugteren Fachpersonen (Beamten) das Feld überlassen. Sie werden nie die volle Verantwortung übernehmen können, von den zusätzlichen Kosten ganz zu schweigen. Ein Gesundheitssystem mit weniger Ärzten wird also nicht besser und auch nicht billiger sein und zusätzlich ist die ärztliche Kompetenz nicht mehr gewährleistet. Letztendlich ist dann niemand mehr für den Patienten zuständig. Der Umgang mit dem kranken Menschen muss individuell bleiben und darf niemals globalisiert erfolgen.

Dass wir Ärzte uns in Zukunft nur auf evidenzbasierte, also statistisch erhärtete, Entscheidungen stützen sollen, ist auch kein Wundermittel für die Lösung aller Probleme, besonders, wenn «evidence based» und gesunder Menschenverstand aufeinanderprallen: Wie beim Fallschirmspringen, wo die rettende Wirkung des Fallschirms noch nie statistisch kontrolliert und bewiesen wurde, wo man aber dessen Gebrauch beim Abspringen dennoch getrost empfehlen kann. (Zitat). Oder wenn für eine onkologische Behandlung ein neues, sehr teures Medikament eingeführt wird, das statistisch nachgewiesen eine $13 \%$ bessere Wirkung zeigt, was für den Krebspatienten wahrscheinlich wenig bringt, für die Industrie jedoch einen millionenschweren 
Gewinn bedeutet und somit auch für das Gesundheitswesen belastend ist. Es wird bei solchen Abwägungen für manche Entscheidungen immer Grauzonen geben, die ein breiteres und nicht nur technisches Erfassen erfordern. Eine Klärung der Probleme mit der TARMED-Mentalität wird da zu nichts führen.

Ganz allgemein werden auch weiterhin unsere Überlegungen nicht evidenzfundiert, sondern auf Grund von Modellvorstellungen erfolgen. Von Modellvorstellungen (Paradigmen) und nicht «evidence based» nehmen auch die wichtigsten Forschungsimpulse ihren Ursprung. Spitzenmedizin ist unerlässlich für einige Patienten und für die weitere Entwicklung der Medizin. Uns allen kann sie aber nicht unbedingt die Angst mindern oder das Leben verlängern. Dazu braucht es eine gute Basismedizin.

\section{Das einzige Kriterium für die Güte einer Therapie ist und bleibt jedoch der Nutzen für den Patienten. Und dieser wiederum hängt direkt von der genauen Diagnose ab.}

Dass wir sparen müssen, ist unbestritten. Diese Tatsache hat zu verschiedener politischer, übergeordneter Zielsetzung geführt, die alles wieder verzerrt und bisher nichts gebracht hat.

\section{Das Hauptopfer ist unsere Medizin, die schlechter wird durch das Sparen am falschen}

Ort.

\section{Gedanken zur Machtverschiebung im neuen Gesundheitswesen}

Der TARMED wurde leider von den Ärzten demokratisch und gutgläubig angenommen, jetzt wird er diktatorisch und manchmal auch mit nicht ganz lauteren Mitteln durchgesetzt, auch wenn der einzige Erfolg es sei («Medical Tribune», Juni 2006), dass er überhaupt eingeführt werden konnte. Der Staat versucht auf Kosten der Ärzte zu sparen, und bereitet so die Verstaatlichung der Medizin vor. Was mit den durch santésuisse bei den Ärzten eingetriebenen Beträgen bisher geschehen ist, darüber herrscht
Stillschweigen: Kommen sie wirklich den Patienten wieder zugute oder dienen sie vor allem dazu, die Herrschaft von santésuisse weiter auszubauen, oder versickern die Gelder im Sand?

Ein Jahr danach - one year after: Man kann es drehen wie man will, wir sind dem beamteten Gesundheitssystem nochmals nähergerückt und das Finale der Verstaatlichung ohne Ersparnis ist in Reichweite. Hier noch einige Beispiele, die unsere gegenwärtige gesundheitspolitische Lage besser beleuchten:

- Das Vorgehen der Syndikate der Arbeiter, die viel helfen könnten, aber im Verkehr mit den Ärzten oft nicht den richtigen Ton finden: Von ihren sozial abgesicherten Angestellten kann einem unabhängigen Arzt heute aus heiterem Himmel gebieterisch befohlen werden, Rapporte und Kopien auszustellen, und zwar gratis, da die Patientin mittellos sei und man ihr daher keine Rechnung stellen könne. Nicht Rechnung stellen ja oder nein ist hier die Frage, sondern, dass es auch heute immer noch dem Arzt überlassen sein muss, ob er Rechnung stellen will oder nicht, und noch wichtiger, dass er bestimmt, ob er auf der Bezahlung bestehen will oder nicht. Es handelt sich hierbei nicht um ein finanzielles, sondern um ein richtungsweisendes, machtpolitisches Problem. Die auf diesen Fall angesprochenen, kantonalen Behörden haben es vorgezogen, nicht auf diese Frage einzutreten.

- Von seiten der Behörden wird auch die Meinung vertreten, dass es schon immer Probleme mit den Ärzten gegeben hat, wobei ihr Ansehen und ihr Einfluss im Abnehmen sind, und dies zum Teil aus eigenem Verschulden: Sie haben nie anderes gemacht als gute Miene zum bösen Spiel. Der Einfluss der Behörden nahm jedoch stetig zu (nicht unbedingt ihr Ansehen). Ärzte als Vollblutpolitiker sind oder wären unentbehrlich und fänden ein weites Betätigungsgebiet, wenn sie die Medizin wirklich verteidigen wollten. Der Mangel an Ärzten dieser Sparte zeigt eben, dass die meisten die Medizin der Medizin halber gewählt haben, und nicht aus anderen Motiven, was für sie spricht.

- Die Suva zielt darauf ab, ihre Menüs weiterhin selbst $\mathrm{zu}$ kochen und $\mathrm{zu}$ verfeinern. Das Resultat: In den letzten Jahren konnte eine zunehmende, sicher gewollte und gelenkte Machterweiterung dieser Versicherung auf Kosten der Ärzte festgestellt werden. Sie lässt uns qualitativ hochstehende, ausführliche und illustrierte Dokumentationen der von ihr selbst veranlassten Untersuchungen an unseren Patienten zukommen, wie wir sie in der 
uns zugestandenen TARMED-Zeit (bezeichnenderweise gerade von ihr selbst konzipiert) niemals mehr selbst so ausführen könnten, wie es früher möglich war. Es ist dies als ein unlauterer Wettbewerb zu betrachten. Gleichzeitig spart die Suva auf unsere Kosten, indem sie stillschweigend unter dem fadenscheinigen, aber einleuchtenden Vorwand, sparen zu müssen, keine Gutachten mehr anfertigen lässt, die ihr früher so wichtig schienen, und die sie offenbar jetzt nicht mehr braucht. Dass ihre hauseigenen Beurteilungen dann nicht immer FMH-gerecht erfolgen, scheint niemanden zu stören und bleibt unwidersprochen. Sie hat sich schon heute eine so starke Position geschaffen, dass sie über eine beträchtliche und unantastbare Eigendynamik verfügt. Hier verdient sie wirklich ihr publizitäres Attribut «mehr als eine Versicherung». Sie ist das Paradebeispiel einer Unfallversicherung auf dem Weg zur machtpolitischen Selbständigkeit.

Neuerdings hat die Suva mit Genugtuung erstmals wieder schwarze Zahlen veröffentlicht. Sie rentiert also wieder - sicher eine grosse Leistung. Was aber mit dem zu vielen Geld geschieht, wurde nicht verlautbart. Kommt es den Prämienzahlern, den Arbeitgebern, den Patienten wieder zugute oder wird es weiter zinsbringend angelegt? Wir kritisieren häufig die Gesundheitssysteme der umgebenden Länder mit dem Unterton der Selbstzufriedenheit, dass wir es in der Schweiz besser machen. Damit ist es momentan nicht mehr gut bestellt. Aus verschiedenen Motiven kam die notwendige Umordnung bei uns nur später, dafür um so rücksichtsloser und mit einem schwer aufliegenden und unverdaulichen TARMED, der ein Buch mit 7 Siegeln bleibt. Wir sind gerade dabei, dieselben Fehler wie unsere Nachbarländer zu wiederholen.

\section{Finanzielle Überlegungen und TARMED}

Im Gegensatz zu uns Ärzten fehlt unserem Gesundheitswesen eine klare, öffentliche Kostenabrechnung über das Kontrollsystem, das der TARMED mit sich bringt, unter Einschluss aller Saläre der damit beschäftigten Funktionäre. Ohne ein solches können aber Input und Output nicht gegeneinander abgewogen werden. Die Transparenz, die von uns Ärzten dringend gefordert wird, fehlt hier ganz.

Die helvetische Medizin macht eine Gratwanderung durch. Der Staat gibt vor zu sparen, würgt aber gleichzeitig die Ärzte, die aufgrund ihrer Ausbildung und Tätigkeit eigentlich Stützpfeiler des Sanitätswesens sein und bleiben sollten. Das entstehende Vakuum soll durch Verstaatlichung kompensiert werden. Trotz anderslautenden Kommentaren kostet diese ganze Übung viel Geld, ohne dass die Öffentlichkeit etwas darüber erfährt. Da der Staat ja immer den längeren Arm hat (haben muss), ist die Machtübernahme ohne grosse Schwierigkeiten erfolgt. Eine Kompetenzübernahme fehlt jedoch und wird es nie geben. Auch ärztliche Verantwortung wird der Staat nie voll übernehmen können. Es ist naheliegend, mit Einschränkungen von Laborabklärungen sparen zu wollen. Wenn zu Beginn einer spezialärztlichen Abklärung aber manchmal etwas mehr als nur unbedingt nötige Blutuntersuchungen vorgenommen werden, können damit oft weitere Konsultationen und andere Untersuchungen vermieden werden, dies ist volkswirtschaftlich und für den Patienten von grossem Vorteil, und sicherlich auch für den Staat. Es sei noch ein weiteres Beispiel aus dem Fachgebiet der Dermatologie genannt: Der DLQI (Dermatologic Life Quality Index) ist in der Tat eine Neuerung. Erstmals wird als Indikation für die Therapie z.B. einer Psoriasis mit neuen, sehr teuren und auf lange Sicht zu verabreichenden Medikamenten (biologics) auch das psychische Leiden des Patienten an seiner Krankheit mitberücksichtigt. Es handelt sich bei der Psoriasis um eine chronische, dermatologische Erkrankung mit Tendenz zu intern-medizinischen Veränderungen und Komplikationen, eine Krankheit, die sicher ein Leben entscheidend verändern kann. Es wird in Zukunft jedoch schwierig sein, bei weiteren sich ergebenden Indikationen das Recht auf Bezahlung festzulegen, da es auch als kosmetisch zu betrachtende Veränderungen zu werten geben wird, die sicher den DLQI stark beeinflussen. Um dies zu beurteilen, wird es eben nicht nur fachliche Kompetenz, sondern vor allem ärztliche Erfahrung brauchen.

\section{Schlussbetrachtung}

Die grundsätzliche Frage, ob es besser ist, wissende (miteingeschlossen Besserwisser) Fachspezialisten als medizinische Berater zu haben, oder doch besser Ärzte, die auch noch zum Ahnen und Fühlen fähig sind [3], ist immer noch unbeantwortet. Für Bundesrat Couchepin muss ein grosser Gelehrter nicht unbedingt auch ein grosser Politiker sein, wenn ihm der Sinn für das Machbare fehlt [5] (wenn er das Machbare nicht erahnt), womit er sicher recht hat. Er beantwortet damit selbst ungewollt auch gleich für uns die Frage, ob es ideal wäre, nur rationale Ärzte zu haben (evidence based), oder solche, die eben wie gute Politiker auch - zusätzlich noch befähigt sind, zu ahnen und zu fühlen (mitzu- 
fühlen), d.h. nicht nur welche, die wissen, was vorgeht, sondern vielmehr solche, die es auch verstehen.

\section{Finale}

In der Oper kommt der Held eines Dramas am Schluss noch um oder er begeht Selbstmord. Zwar wird hier kein Held getötet und niemand begeht Selbstmord, doch wird ein alter Arzt vom Staat entgegen aller Abmachungen seit über 2 Jahren (keine Antwort ist auch eine Antwort) in seiner Dignität verletzt [6]. (Nichtberücksichtigung und Nichteinbeziehung von drei FMHTiteln). Unsere jetzige Regierung scheut offensichtlich auch vor grundlegend unschweizerischen Massnahmen nicht zurück, um sich zu bestätigen.

Im Bühnenbild des Schlussaktes dieses helvetischen Trauerspiels wird ein mit Kies überladenes, dahintreibendes, altes Ledischiff der Reederei «Suvbagtar SA.suisse» gezeigt, einer eidgenössischen (Kon)Fusionsgesellschaft, die sich aufs Baggern spezialisiert und sich damit einen Namen geschaffen hat. Der Himmel ist verdunkelt von geballten Kumuluswolken, das grüne Wasser tobt und schäumt. In der näheren Ferne ragen schwer auszumachende, grauschwarze Klippen drohend aus dem Wasser. Wir befinden uns wahrscheinlich auf dem Urnersee gegenüber von Flüelen in Richtung Attinghausen und das Schiff driftet stetig auf die Felsen zu. Man hört im Hintergrund dumpfe Musik mit verhaltenen, tiefen Paukenschlägen - wie nicht allzuferner Donner. Und über dieses Finale senkt sich langsam der schwere, rote Vorhang. Man fragt sich, ob der Tell wohl noch einmal springen wird? (ohne TARMED!) «Ich sah's mit Augen an, ihr könnt mir's glauben,
s'ist alles so geschehen, wie ich Euch sagte.»

(Aus dem Schauspiel «Wilhelm Tell» von Friedrich Schiller.)

\section{Literatur}

1 Radio Suisse Romande: www.rsr.ch/les-infos/virus/.

2 Hugentobler W. Kostenvergleich ... Primary Care. 2006;6:586-9.

3 Mumenthaler M. Medizinstudium und Arztberuf. Schweiz Ärztezeitung. 2007;88(1):18-21.

4 Hasler N. Einkommensverhältnisse der freien Ärzteschaft der Schweiz in den Jahren 2003 (neu) und 2002 (Re-Evaluation). Vollerhebung im Juni 2006. Schweiz Ärztezeitung. 2006;87(39):1677-86.

5 Widmer D. UEMO gegen Couchepin. Primary Care. 2006;6:566-9.

6 Dignität: gemäss Duden: persönliche Würde. Ist auch ein im TARMED verankerter Begriff, der auf Grund seiner von der FMH verbürgten Ausbildung jedem Arzt die Tätigkeit, die bewilligten Tarifpositionen und seinen sich daraus ergebenden Falldurchschnitt zuordnen sollte.

Für die sprachliche Durchsicht danke ich folgenden Personen:

- deutsch: Herrn Prof. Emil Steinhäuser, Erlangen;

- français: Dr. André Bodmer, Nyon, et Dr. Fabrice Vust, Neuchâtel;

- italiano: Dott. Saverio Prinz, Camorino, Sig.ra Graziella Ghisalberti-Berri, San Vittore.

Veröffentlichung auf italienisch in der TMT. 